\title{
A System for Monitoring and Tracking the LHC Beam Spot within the ATLAS High Level Trigger
}

\author{
R. Bartoldus ${ }^{a}$, C. Bee ${ }^{b}$, J. Cogan $^{a}$, A. Salnikov $^{a}$, E. Strauss ${ }^{a}$, F. Winklmeier $^{c}$ \\ On behalf of the ATLAS Collaboration
}

\section{Abstrac}

The position, size and orientation of the luminous region of the beam spot produced by the LHC in the ATLAS interaction region are computed online using the ATLAS High Level Trigger (HLT) system. The high rate of triggered events is exploited to make precise measurements of the parameters in near real-time, as they change significantly even during a single data-taking run. We present the challenges for online determination and monitoring of the beam spot in ATLAS and a feedback system to publish the values for use in the HLT. Results using this system during ATLAS operations in the course of the $2011 \mathrm{LHC}$ run at $\sqrt{\boldsymbol{s}}=\mathbf{7}$ TeV and the 2012 run at $\sqrt{s}=8 \mathrm{TeV}$ are shown. A specially designed algorithm, which uses tracks registered in the silicon detectors to reconstruct event vertices, is executed on the HLT processor farm of several thousand CPU cores. Monitoring histograms from all the cores are sampled and aggregated across the farm every 60 seconds. The reconstructed beam values are corrected for detector resolution effects, measured in situ from the separation of vertices whose tracks have been split into two collections. Furthermore, measurements for individual bunch crossings have allowed for studies of single-bunch distributions as well as the behavior of bunch trains, calibrated to the beam average. Run control invokes a comparison of the nominal and measured beam spot values, and when threshold conditions are satisfied the farm configuration is updated. To achieve sharp time boundaries across the event stream, which is triggered at rates of several $\mathrm{kHz}$, a special datagram is injected into the event path via the Central Trigger Processor that signals the pending update to the trigger nodes. Thousands of clients then fetch the same set of values from the conditions database in a fraction of a second via an efficient, nearly-simultaneous access made possible through a dedicated CORAL Server and Proxy tree.

\section{Event Vertex Distributions}

Online Selection

- The Level 2 Trigger vertexing and beam spot measurements are initiated by signals from a Level 1 multi-jet trigger.

- Data collection and pattern recognition on the entire Inner Detector must be done within only a few tens of milliseconds.

- A fast vertex fitter reconstructs primary vertices with $\geq 4$ tracks of $p_{\mathrm{T}}>1 \mathrm{GeV}$ and $|\eta|<2.5$.

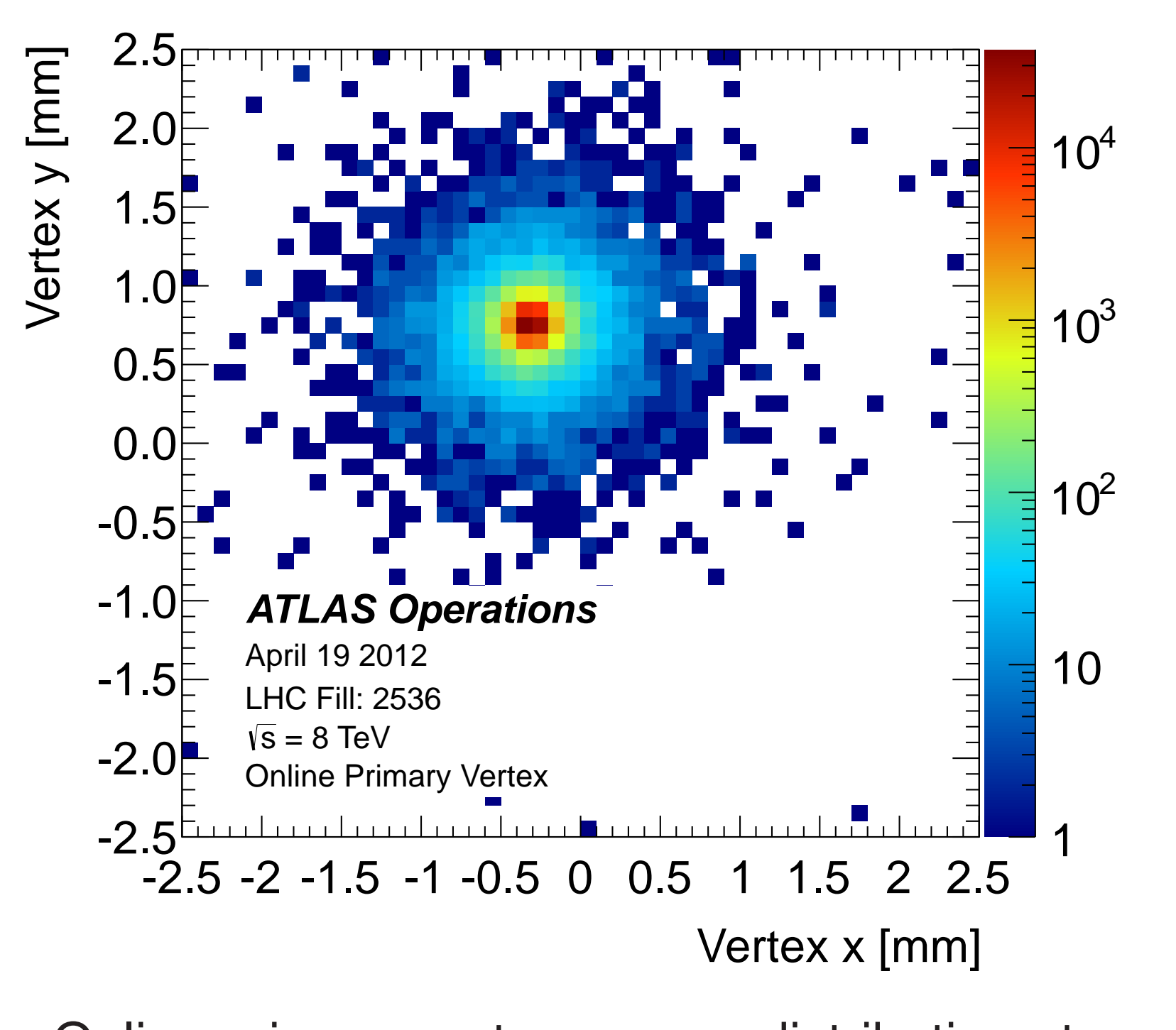

Online primary-vertex $x$ vs. $y$ distribution at $\sqrt{s}=8 \mathrm{TeV}$
Online Monitoring

- Distribution of vertices is characterized very precisely due to the high rate of events.

- The time evolution of the position, widths and tilts is recorded in approximately 2-minute intervals.

- Upon significant changes, new values are pushed to the conditions database for use by the trigger farm.

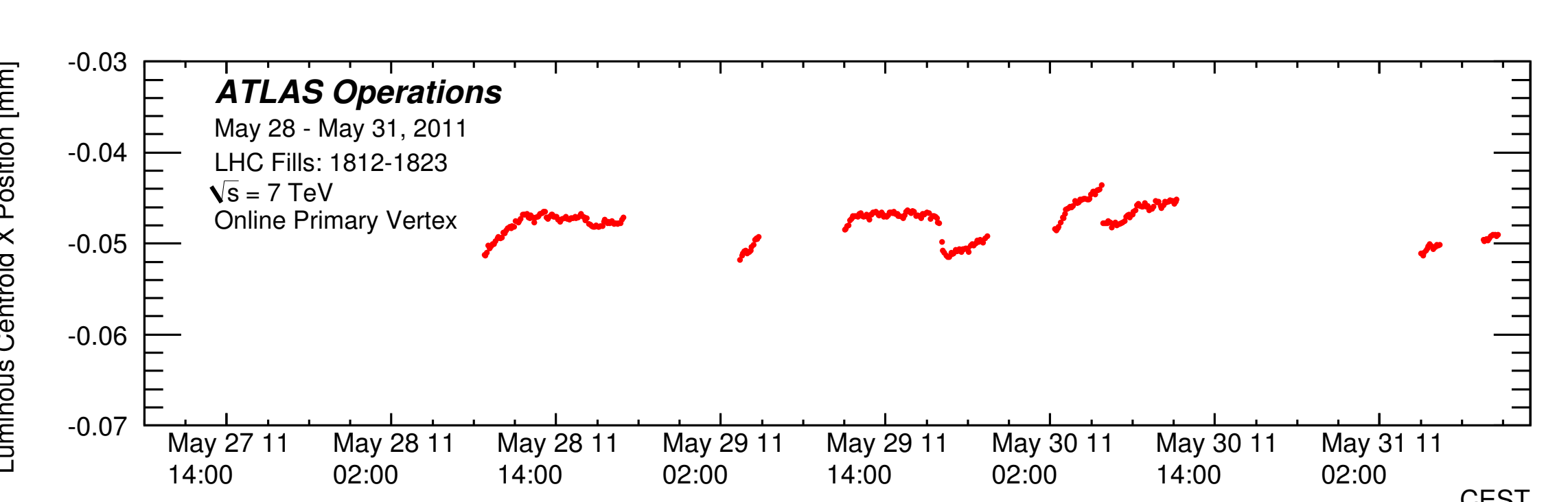

Evolution of the absolute beamspot position.

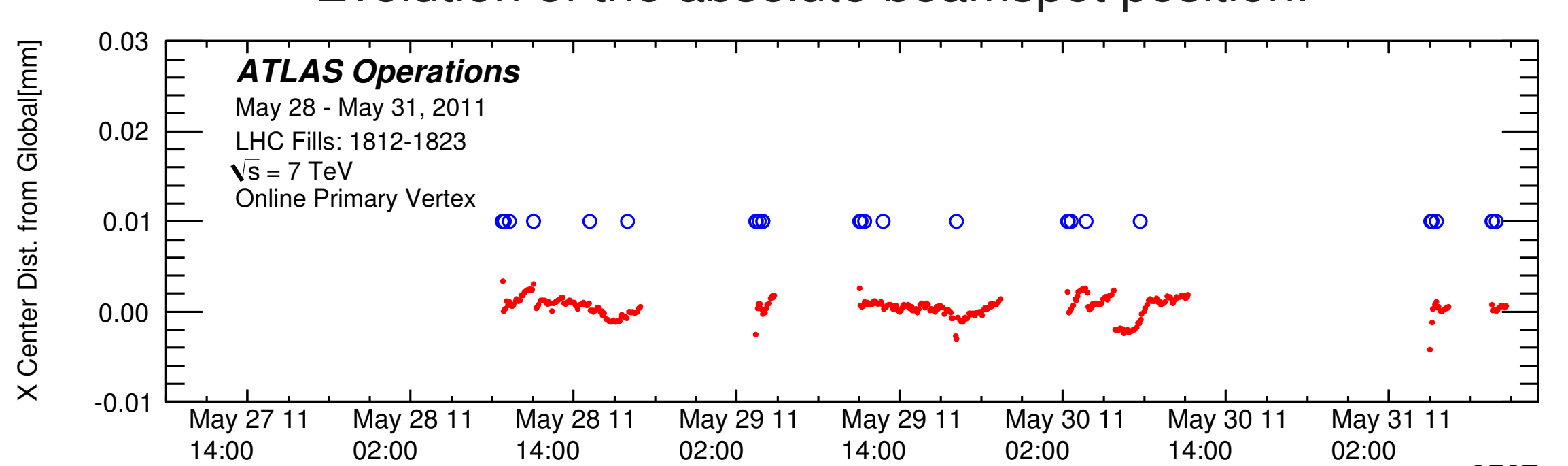

Evolution of the beamspot position relative to the last value stored to the conditions database (red) and the update intervals (blue).

\section{Elements Overview}

HLT

- High Level Trigger

- Second (software) stage of two-level trigger system

- Farm of currrently $1600 \mathrm{HLT}$ nodes with 17000 cores in total

- No cross-processor

communication

- Requests data fragments from CTP and detector Read-Out Systems (ROS), e.g. Pixel Silicon

\section{Oracle}

- Back-end technology powering

a conditions database

- Makes use of a COOL

abstraction layer

\section{CORAL Server}

- Translates between CORAL and Oracle commands

Proxy Tree

- Efficient database querying and caching between the HLT and the conditions database

- Each non-leaf node caches requests and propagates new ones up

Pixel and SCT ROS

- Silicon Tracker Read-Out Systems

- Provide subdetector data reception

- Handle buffering and distribution

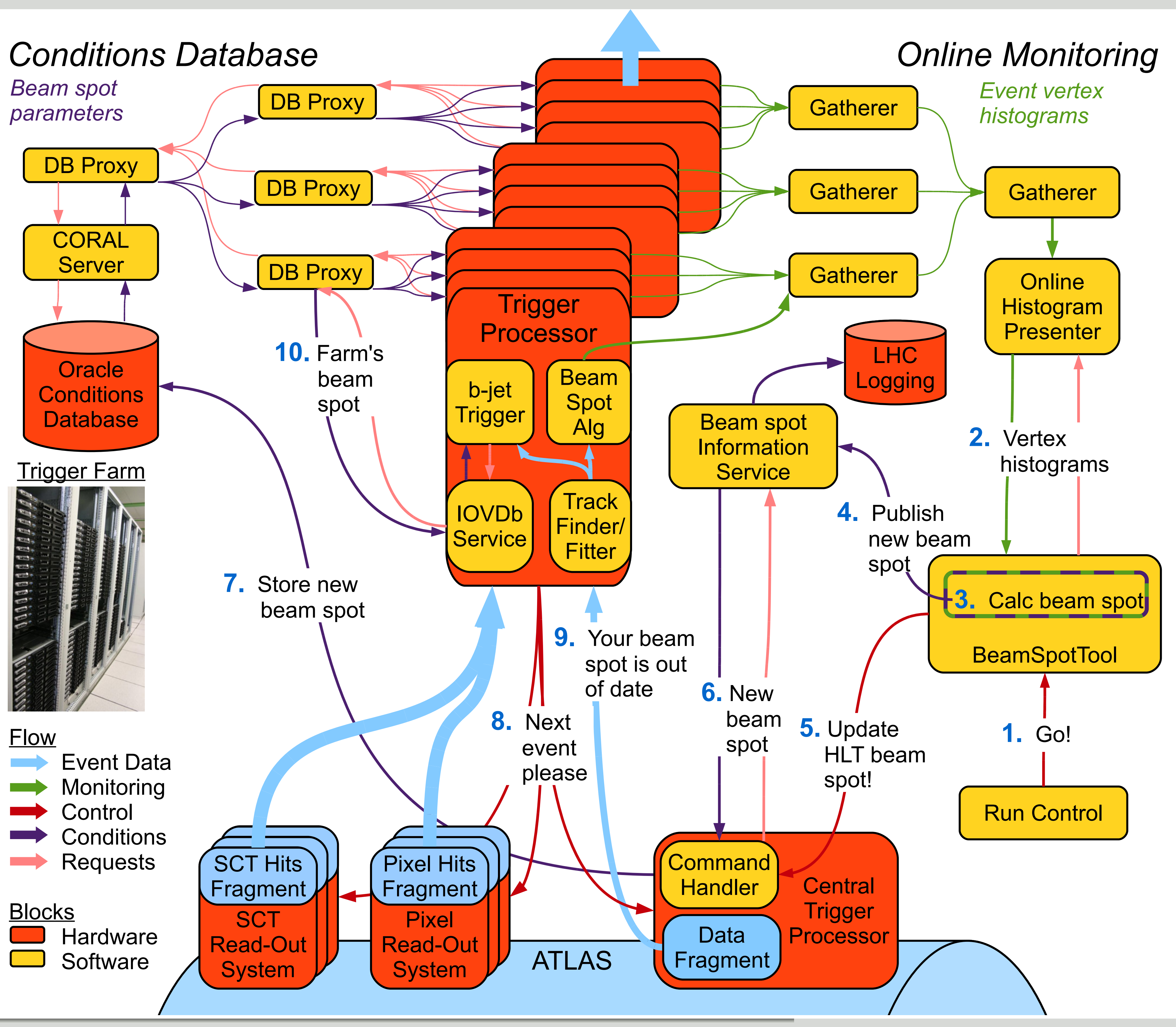

\section{CORAL Server Proxy}

- Provides scalable solution for 10000 s of clients

- Uses technology-independent protocol optimized for multiplexing and caching

- Multiplexes many client connection into single server connection

- Caches requests in memory to reduce repeated requests

- Supports hierarchical multi-level proxy trees

\section{Elements Overview}

Run Control

- Controls ATLAS finite state machine

- Top-level interface to DAQ operator

\section{CTP}

- Central Trigger Processor

- Generates global L1 accept

- Ships the CTP data fragment

with every accepted event

- Fragment contains flag

indicating beamspot updates to HLT processes

L2SV

- Level 2 Supervisor

- Assigns events to L2 processes - Monitors event counts and rates

IOVDb Service

- Interval of Validity Database Service

- Maintains current conditions data in process memory

\section{Gatherer Tree}

- Collects, adds, and publishes summed histograms

IS

- Online Information Service

- Transient system, reads and broadcasts data

- Used to retrieve the monitoring and $\mathrm{DQ}$ data
- Fetch and aggregate monitoring histograms

- Fit projections of vertex and split-vertex distributions

- Calculate summary beamspot values, estimate and correct for resolution effects

- Publish new values

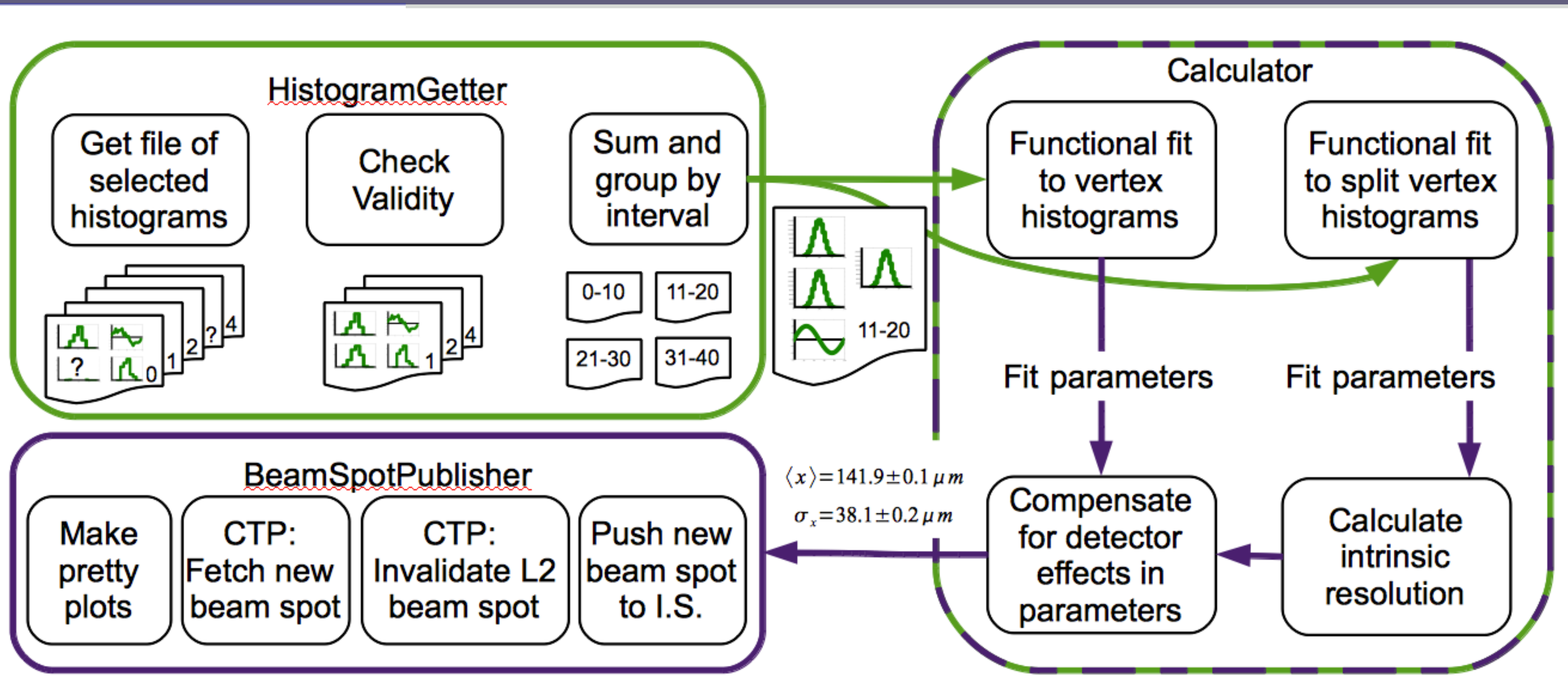

LHC Configuration Page

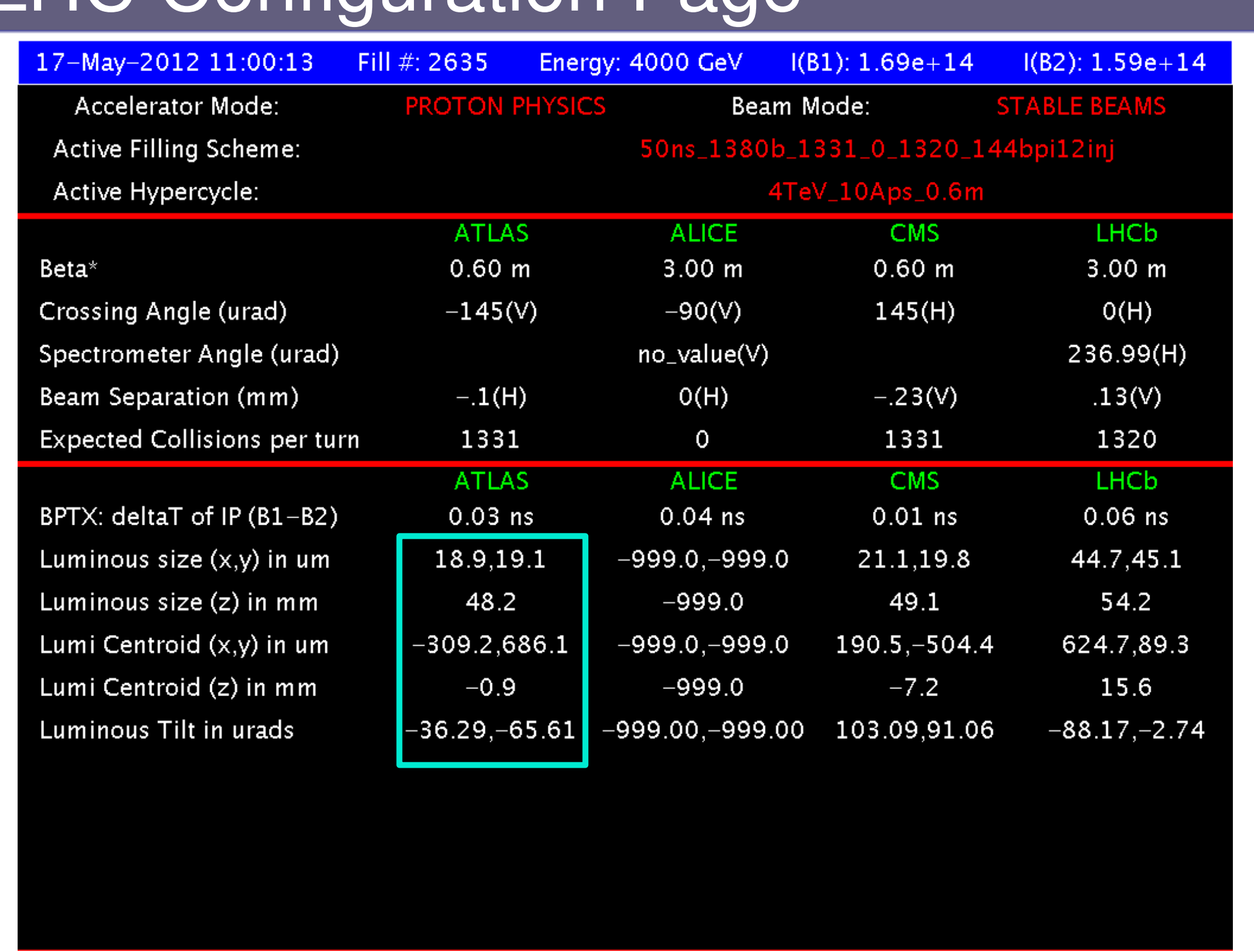

